\title{
ZETA-FUNCTIONS OF HARMONIC THETA-SERIES AND PRIME NUMBERS
}

\author{
A. ANDRIANOV
}

\begin{abstract}
The problem of finding Euler product expansions is treated for zetafunctions of modular forms in one variable that are presented by harmonic thetaseries. On the basis of the author's formulas obtained earlier for the action of the Hecke operators on harmonic theta-functions, Euler product expansions are obtained for eigenfunctions of Hecke operators. For the theta-series of quadratic forms proportional to the sum of two squares, the eigenfunctions of Hecke operators are constructed and the associated Euler expansions are calculated.
\end{abstract}

\section{§1. PRIME NUmbers AND THETA-SERIES}

Concerning rational prime numbers in various arithmetical sequences, it may be noted that no essential progress has been achieved for more than century and a half, since the famous Dirichlet theorem on prime numbers in arithmetic progressions (1837). Still absolutely mystical is the question about prime numbers in quadratic sequences, i.e., about prime numbers of the form $a n^{2}+b n+c$, where $a, b, c$ are rational coprime integers, and $d=b^{2}-4 a c$ is not a rational square. The situation has not been changing despite considerable progress of the algebro-analytic theory of integral quadratic forms after Dirichlet. Our purpose here is to draw the attention of number theorists to some analytic aspects of the theory of quadratic forms possibly related to the problem.

In order to be more specific, we start with the celebrated problem on prime numbers of the form $1+n^{2}$. It is well known that this problem is closely related to reduction over prime modules of certain elliptic curves with complex multiplications by Gauss integers $a+\sqrt{-1} b$, say, of the curve

$$
y^{2}=x\left(x^{2}-1\right) \text {. }
$$

Indeed, in accordance with a formula of D. S. Gorshkov (see [9, Chapter V, Question 8c]), the decomposition of a prime number $p$ of the form $4 k+1$ into a sum of two squares of integers can be written with help of the Legendre symbol as

$$
p=\left(\frac{1}{2} \sum_{x=0}^{p-1}\left(\frac{x\left(x^{2}-1\right)}{p}\right)\right)^{2}+\left(\frac{1}{2} \sum_{x=0}^{p-1}\left(\frac{x\left(x^{2}-b\right)}{p}\right)\right)^{2}
$$

where $b$ is a quadratic nonresidue modulo $p$. It is easily seen that the first square in this decomposition is odd, while the second is even. Hence, a prime $p \equiv 1(\bmod 4)$ has the form $1+n^{2}$ if and only if

$$
\frac{1}{2} \sum_{x=0}^{p-1}\left(\frac{x\left(x^{2}-1\right)}{p}\right)= \pm 1
$$

2010 Mathematics Subject Classification. Primary 11F27; Secondary 11F46, 11F60, 14G10, $20 \mathrm{C} 08$.

Key words and phrases. Euler products, harmonic theta-functions and theta-series, Hecke operators, prime numbers, zeta-functions of theta-functions and theta-series.

The author was supported in part by RFBR (grant 08-01-00233). 
It is well known (see, e.g., [10]) that the points of the projective closure of the affine elliptic curve (1.1) rational over the field of $p$ elements form a finite Abelian group of the order

$$
N_{p}=p+1+\sum_{x=0}^{p-1}\left(\frac{x\left(x^{2}-1\right)}{p}\right),
$$

and so $p$ has the form $1+n^{2}$ if and only if $N_{p}=p+1 \pm 2$. Unfortunately, for the time being the details of the behavior of the numbers $N_{p}$ for different primes $p$ is an open question, and there is no much hope to approach the problem from this side.

By the way, the criterion (1.2) can be reformulated quite elementarily in terms of simple congruences modulo $p$ for appropriate factorials: the well-known properties of the Legendre symbol imply that

$$
\sum_{x=0}^{p-1}\left(\frac{x\left(x^{2}-1\right)}{p}\right) \equiv \sum_{x=0}^{p-1}\left(x\left(x^{2}-1\right)\right)^{\frac{p-1}{2}} \equiv \sum_{x=1}^{p-1} x^{\frac{p-1}{2}}\left(x^{2}-1\right)^{\frac{p-1}{2}} \equiv-\left(\begin{array}{c}
\frac{p-1}{2} \\
\frac{p-1}{4}
\end{array}\right) \quad(\bmod p),
$$

so that the criterion (1.2) is equivalent to the congruence

$$
-\frac{1}{2}\left(\frac{\frac{p-1}{2}}{\frac{p-1}{4}}\right)=-\frac{1}{2} \frac{\left(\frac{p-1}{2}\right) !}{\left[\left(\frac{p-1}{4}\right) !\right]^{2}} \equiv \pm 1 \quad(\bmod p),
$$

which can be written in the form

$$
4\left[\left(\frac{p-1}{4}\right) !\right]^{4} \equiv-1 \quad(\bmod p) .
$$

This looks nice, but seems to be out of use for the problem of primes in the sequence $1+n^{2}$, like the Wilson theorem does not help to prove that there are infinitely many primes.

Fortunately, our problem is closely related not only to reduction of elliptic curves modulo prime numbers or to congruences for factorials, but also to such a powerful tool for the study of quadratic forms as modular forms for subgroups of the modular group $\Gamma=S L_{2}(\mathbb{Z})$. Consider the function defined on the upper half-plane of the complex variable

$$
\mathbb{H}=\{z=x+i y \in \mathbb{C} \mid y>0\} \quad(i=\sqrt{-1})
$$

by a harmonic theta-series of the quadratic form $\mathbf{q}=8\left(x_{1}^{2}+x_{2}^{2}\right)$ (of the level $l=32$ ) (see $\S 2)$ :

$$
\begin{aligned}
F(z) & =4 \sum_{n_{1}, n_{2} \in \mathbb{Z}}\left(n_{2}+\frac{1}{4}\right) e^{\pi i z 16\left(\left(n_{1}+\frac{1}{4}\right)^{2}+\left(n_{2}+\frac{1}{4}\right)^{2}\right)} \\
& =\sum_{\substack{m_{1}, m_{2} \in \mathbb{Z}, m_{1} \equiv m_{2} \equiv 1}} m_{2} e^{\pi i z\left(m_{1}^{2}+m_{2}^{2}\right)}=\sum_{n=1}^{\infty} c(n) e^{2 \pi i z n}
\end{aligned}
$$

where

$$
c(n)=\sum_{\substack{m_{1}, m_{2} \in \mathbb{Z}, m_{1}^{2}+m_{2}^{2}=2 n \\ m_{1} \equiv m_{2} \equiv 1}} m_{2}
$$

are the Fourier coefficients of $F$. Clearly, these coefficients satisfy the relations $c(1)=1$, and $c(n)=0$ unless $n \equiv 1(\bmod 4)$. In particular, $c(p)=0$ if $p$ is a prime number of the form $4 k+3$, but if $p$ is a prime number of the form $4 k+1$ and $\left(a_{1}, a_{2}\right)$ is one of the integral solutions of the equation $x_{1}^{2}+x_{2}^{2}=p$, then all integral solutions of the equation are $\left( \pm a_{1}, \pm a_{2}\right)$ or $\left( \pm a_{2}, \pm a_{1}\right)$; hence, all integral solutions of the equation $y_{1}^{2}+y_{2}^{2}=2 p$ 
are $\left( \pm\left(a_{1}-a_{2}\right), \pm\left(a_{1}+a_{2}\right)\right)$ or $\left( \pm\left(a_{1}+a_{2}\right), \pm\left(a_{1}-a_{2}\right)\right)$. Since, clearly, it can be assumed that $a_{1} \equiv 1(\bmod 4)$ and $a_{2}$ is even, from (1.6) it follows that

$$
c(p)=\left\{\begin{array}{lll}
\left(a_{1}+a_{2}\right)+\left(a_{1}-a_{2}\right)=2 a_{1} & \text { if } a_{2} \equiv 0 \quad(\bmod 4), \\
\left(-a_{1}+a_{2}\right)+\left(-a_{1}-a_{2}\right)=-2 a_{1} & \text { if } a_{2} \equiv 2 \quad(\bmod 4) .
\end{array}\right.
$$

In any case, a prime number $p$ of the form $4 k+1$ has the form $1+n^{2}$ if and only if

$$
c(p)= \pm 2 \text {. }
$$

The series (1.5) converges absolutely on $\mathbb{H}$ and uniformly on compact subsets of $\mathbb{H}$. Consequently, this series determines a holomorphic function on $\mathbb{H}$. It is known that the function $F$ is a modular (cusp) form of weight 2 for the congruence subgroup

$$
\Gamma_{0}(32)=\left\{\left(\begin{array}{ll}
\alpha & \beta \\
\gamma & \delta
\end{array}\right) \in S L_{2}(\mathbb{Z}) \mid \gamma \equiv 0 \quad(\bmod 32)\right\}
$$

of the modular group $\Gamma=S L_{2}(\mathbb{Z})$ (see, e.g., [7] or [6, Theorem 20]).

The problem about prime numbers satisfying condition (1.7) looks largely like the problem about prime numbers $p$ with a given value $\chi(p)$ of a Dirichlet character $\chi$, and the latter is closely related to the question concerning prime numbers in arithmetic progressions. In the same way as the problem on the values of characters at prime numbers cannot be approached in terms of an individual character but requires all characters of a given module, the problem of separating the prime numbers $p$ with given value of the coefficient $c(p)$ should perhaps be considered in a wider context of similar problems for other harmonic theta-series. In this paper we start to move in this direction.

Note, by the way, that the problem about prime numbers of the form $1+n^{2}$ is closely related to the problem of twins in the ring $\mathcal{O}=\mathbb{Z}[i]$ of Gauss integers, because if a prime $p$ has the form $1+n^{2}$, then the numbers $i n-1$ and $i n+1=(i n-1)+2$ are prime twins in the ring $\mathcal{O}$, although, obviously, this ring contains no rational prime twins. Possibly, this hints that the classical problem of prime twins for the ring of rational integers has also a quadratic nature.

Contents of the paper. In $\S 2$ we recall the basic definitions and facts about harmonic theta-functions together with their zeta-functions. In $\S 3$ we consider the action of Hecke operators on theta-functions. $\S 4$ is devoted to the action of (hereditary) Hecke operators on theta-series and the deduction of the corresponding Euler products. In $\S 5$ we analyze an example of harmonic theta-series and zeta-functions for multiples of the sum of two squares.

Notation. As usual, we fix the letters $\mathbb{N}, \mathbb{Z}, \mathbb{Q}, \mathbb{R}$, and $\mathbb{C}$ for the set of positive rational integers, the ring of rational integers, the field of rational numbers, the field of real numbers, and the field of complex numbers, respectively.

$\mathbb{A}_{n}^{m}$ denotes the set of all $(m \times n)$-matrices with elements in $\mathbb{A}$. If $\mathbb{A}$ is a ring with the identity element, $1_{n}=\mathbf{1}$ and $0_{n}=\mathbf{0}$ denote the identity element and the zero element of $\mathbb{A}_{n}^{n}$, respectively. The transpose of a matrix $M$ is always denoted by ${ }^{t} M$. For two matrices $A$ and $B$ of appropriate size we write

$$
A[B]={ }^{t} B A B .
$$

\section{§2. ZETA-FUNCTIONS OF HARMONIC THETA-FUNCTIONS}

In this section, we recall the definitions of the harmonic theta-functions and the corresponding zeta-functions. 
Let

$$
\mathbf{q}(X)=\sum_{1 \leq \alpha \leq \beta \leq m} q_{\alpha \beta} x_{\alpha} x_{\beta}=\frac{1}{2}{ }^{t} X Q X=\frac{1}{2} Q[X] \quad\left({ }^{t} X=\left(x_{1}, \ldots, x_{m}\right)\right)
$$

be a real positive definite quadratic form in $m$ variables with the matrix

$$
Q=Q(\mathbf{q})=\left(q_{\alpha \beta}\right)+{ }^{t}\left(q_{\alpha \beta}\right) .
$$

Since the form $\mathbf{q}$ is real and positive definite, there exists is a real matrix $S$ such that $Q(\mathbf{q})={ }^{t} S S$. A homogeneous polynomial of degree $g$ in $x_{1}, \ldots, x_{m}$ of the form

$$
P(X)=P_{Q}(X)=P_{0}(S X),
$$

where $P_{0}=P_{0}(X)$ is a homogeneous polynomial of degree $g$ in $x_{1}, \ldots, x_{m}$ satisfying the Laplace equation

$$
\sum_{1 \leq \alpha \leq m} \frac{\partial^{2} P_{0}(X)}{\left(\partial x_{\alpha}\right)^{2}}=0
$$

is called a harmonic polynomial of degree $g$ with respect to the form $\mathbf{q}$. The definition can easily be reformulated as follows: a homogeneous polynomial $P$ of degree $g$ in $x_{1}, \ldots, x_{m}$ is harmonic with respect to the form $\mathbf{q}$ with matrix $Q$ if it satisfies the differential equation

$$
\sum_{1 \leq \alpha, \beta \leq m}\left(Q^{-1}\right)_{\alpha \beta} \frac{\partial^{2} P(X)}{\partial x_{\alpha} \partial x_{\beta}}=0
$$

It can be verified that a polynomial of the form

$$
P(X)=\left({ }^{t} \Omega Q X\right)^{g},
$$

where $\Omega \in \mathbb{C}^{m}$ is an isotropic vector of the form $\mathbf{q}$ with the matrix $Q$, i.e., a complex $m$-vector satisfying

$$
\mathbf{q}(\Omega)=\frac{1}{2}{ }^{t} \Omega Q \Omega=0,
$$

is a harmonic polynomial of order $g$ with respect to $\mathbf{q}$, and each harmonic polynomial of order $g$ with respect to $\mathbf{q}$ is a finite sum of such polynomials.

For a real positive definite quadratic form $\mathbf{q}$ in $m$ variables with matrix $Q$ and a harmonic polynomial $P$ of order $g$ with respect to $\mathbf{q}$, the theta-function of $\mathbf{q}$ (of genus one) with harmonic polynomial $P$ and parameters $(U, V)$, where $U={ }^{t}\left(u_{1}, \ldots, u_{m}\right), V=$ ${ }^{t}\left(v_{1}, \ldots, v_{m}\right) \in \mathbb{C}^{m}$, is defined by the series

$$
\Theta_{P}(z ; Q,(U, V))=\sum_{N \in \mathbb{Z}^{m}} P(N-V) e^{\pi i\left(z Q[N-V]+2 \cdot{ }^{t} U Q N-{ }^{t} U Q V\right)},
$$

where $z=x+i y \in \mathbb{H}$. This series converges absolutely for $z$ in the upper half-plane and converges uniformly in each half-plane $\{z \in \mathbb{C} \mid \operatorname{Im} z \geq \epsilon\}$ with $\epsilon>0$.

In accordance with a specialization of the general inversion formula, see [2, Lemma 5.1], the theta-function (2.2) satisfies the following inversion formula:

$$
\Theta_{P}(-1 / z ; Q,(V,-U))=\frac{i^{m / 2}}{\sqrt{\operatorname{det} Q}}(-z)^{\frac{m}{2}+g} \Theta_{P^{*}}\left(z ; Q^{-1}, Q(U, V)\right),
$$

where

$$
P^{*}(X)=P\left(Q^{-1} X\right)
$$

is a harmonic polynomial of degree $g$ with respect to the form $\mathbf{q}^{*}$ and with matrix $Q^{-1}$.

In order to define the zeta-function associated with the harmonic theta-function (2.2), we use the Euler integral

$$
\int_{0}^{\infty} y^{s-1} e^{-\alpha y} d y=\Gamma(s) \alpha^{-s} \quad(\alpha>0, \operatorname{Re} s>0)
$$


where $\Gamma(s)$ is the gamma-function. For $\operatorname{Re} s>\frac{m}{2}$ we obtain

$$
\begin{aligned}
\int_{0}^{\infty} & y^{s+g / 2-1}\left(\Theta_{P}(i y ; Q,(U, V))-e^{\pi i^{t} U Q V} \rho(V, g)\right) d y \\
& =\sum_{N \in \mathbb{Z}^{m}, N \neq V} P(N-V) e^{2 \pi i^{t} U Q N-\pi i^{t} U Q V} \int_{0}^{\infty} y^{s+g / 2-1} e^{-\pi y Q[N-V]} d y \\
& =(2 \pi)^{-(s+g / 2)} \Gamma(s+g / 2) e^{-\pi i^{t} U Q V} \sum_{N \in \mathbb{Z}^{m}, N \neq V} e^{2 \pi i^{t} U Q N} \frac{P(N-V)}{\mathbf{q}(N-V)^{s+g / 2}}
\end{aligned}
$$

where

$$
\rho(V, g)= \begin{cases}0 & \text { if } V \notin \mathbb{Z}^{m} \text { or } V \in \mathbb{Z}^{m} \text { and } g>0, \\ P(\mathbf{0}) & \text { if } V \in \mathbb{Z}^{m} \text { and } g=0,\end{cases}
$$

and $\mathbf{q}$ is the quadratic form (2.1). The function

$$
\zeta_{P}(s ; Q,(U, V))=\sum_{N \in \mathbb{Z}^{m}, N \neq V} e^{2 \pi i^{t} U Q N} \frac{P(N-V)}{\mathbf{q}(N-V)^{s+g / 2}}
$$

is called the (Epstein) zeta-function of $\mathbf{q}$ with harmonic polynomial $P$ and parameters $(U, V)$. The zeta function converges absolutely for Re $s>m / 2$ and converges uniformly in every half-plane $\operatorname{Re} s>m / 2+\epsilon$ with $\epsilon>0$. Thus, the zeta-function is an analytic function for $\operatorname{Re} s>m / 2$.

The study of analytic continuation and the functional equation for the zeta-function is based on Riemann's method of deducing the analytic properties of zeta-functions from the integral representation and the theta-inversion formula (2.3). After standard considerations, this leads to the following theorem (see, e.g., Siegel's Tata lectures 8 , Chapter I, $\S 5])$.

Theorem 1. The zeta-function $\zeta_{P}(s ; Q,(U, V))$ of a real positive definite quadratic form $\mathbf{q}$ in $m$ variables with a harmonic polynomial $P$ of degree $g$ and parameters $(U, V)$ admits an analytic extension to the whole $s$-plane, which is an entire function of $s$ if either $g>0$ or $g=0$ and the vector $Q U$ is not integral. If $g=0$ and $Q U$ is integral, then the zetafunction is meromorphic in the entire $s$-plane with the only singularity at $s=m / 2$ where it has a simple pole with residue $(2 \pi)^{m / 2} / \sqrt{\operatorname{det} Q} \Gamma(m / 2)$. In all cases $\zeta_{P}(s ; Q,(U, V))$ satisfies the functional equation

$$
\begin{aligned}
& (2 \pi)^{-s} \Gamma(s+g / 2) e^{-\pi i^{t} U Q V} \zeta_{P}(s ; Q,(U, V)) \\
& \quad=\frac{i^{g}}{\sqrt{\operatorname{det} Q}}(2 \pi)^{s-m / 2} \Gamma((m+g) / 2-s) e^{\pi i^{t} U Q V} \zeta_{P^{*}}\left(m / 2-s ; Q^{-1},(-Q V, Q U)\right),
\end{aligned}
$$

where $P^{*}(X)=P\left(Q^{-1} X\right)$.

\section{§3. HECKE OPERATORS}

Starting with this section, we assume that a positive definite quadratic form $\mathbf{q}$ in $m$ variables is integral, i.e., it has rational integral coefficients, in which case the matrix $Q$ of $\mathbf{q}$ is even, i.e., has integral coefficients and even coefficients on the principal diagonal. The smallest number $l \in \mathbb{N}$ such that the matrix $l Q^{-1}$ is even is called the level of the form $\mathbf{q}$. As a particular case of [2, Theorems 4.2-4.3], we obtain the following transformation formulas for the theta-function (2.2) of an integral positive definite quadratic form of level $l$ : for each matrix

$$
\sigma=\left(\begin{array}{ll}
\alpha & \beta \\
\gamma & \delta
\end{array}\right) \in \Gamma_{0}(l)=\left\{\left(\begin{array}{ll}
\alpha & \beta \\
\gamma & \delta
\end{array}\right) \in S L_{2}(\mathbb{Z}) \mid \gamma \equiv 0 \quad(\bmod l)\right\}
$$


the theta-function satisfies the functional equation

$$
\Theta_{P}\left(\frac{\alpha z+\beta}{\gamma z+\delta} ; Q,(U, V)^{t} \sigma\right)=\mu_{\mathbf{q}}(\sigma)(\gamma z+\delta)^{\frac{m}{2}+g} \Theta_{P}(z ; Q,(U, V)),
$$

where $\mu_{\mathbf{q}}(\sigma)$ is an 8th root of unity, which is equal to 1 if $l=1$, but if $l>1$ and $m$ is even, then

$$
\mu_{\mathbf{q}}(\sigma)=\mu_{\mathbf{q}}\left(\left(\begin{array}{ll}
\alpha & \beta \\
\gamma & \delta
\end{array}\right)\right)=\chi_{\mathbf{q}}(\delta)
$$

with the character $\chi_{\mathbf{q}}$ of the quadratic form $\mathbf{q}$, i.e., a real Dirichlet character modulo $l$ satisfying the conditions

$$
\begin{aligned}
\chi_{\mathbf{q}}(-1) & =(-1)^{m / 2}, \\
\chi_{\mathbf{q}}(p) & =\left(\frac{(-1)^{m / 2} \operatorname{det} Q}{p}\right) \quad(\text { the Legendre symbol })
\end{aligned}
$$

if $p$ is an odd prime not dividing $l$, and

$$
\chi_{\mathbf{q}}(2)=2^{-m / 2} \sum_{R \in \mathbb{Z}^{m} / 2 \mathbb{Z}^{m}} e^{\pi i Q[R] / 2}
$$

if $l$ is odd.

In order to approach the natural question on Euler product factorization for zeta functions, we recall the basic definitions and the simplest properties of the (regular) Hecke-Shimura rings and Hecke operators for the groups $\Gamma_{0}(l)$ that appeared above as transformation groups of theta-functions. We follow the general pattern of the theory of Hecke operators on modular forms (see, e.g., [1, Chapter 4], or [3, §2]) in the particular case of the genus $n$ equal to 1 .

We denote by

$$
\mathcal{H}_{0}(l)=\mathcal{H}\left(\Gamma_{0}(l), \Sigma_{0}(l)\right)
$$

the Hecke-Shimura ring of the semigroup

$$
\Sigma_{0}(l)=\left\{M=\left(\begin{array}{ll}
a & b \\
c & d
\end{array}\right) \in \mathbb{Z}_{2}^{2} \mid \operatorname{det} M>0, \operatorname{gcd}(\operatorname{det} M, l)=1, c \equiv 0 \quad(\bmod l)\right\}
$$

relative to the group $\Gamma_{0}(l)$ (over $\mathbb{C}$ ). The ring $\mathcal{H}_{0}(l)$ consists of all formal finite linear combinations with complex coefficients of the symbols $\tau(M)$, which are in one-to-one correspondence with the double cosets $\Gamma_{0}(l) M \Gamma_{0}(l) \subset \Sigma_{0}(l)$. It is convenient to write each of the symbols $\tau(M)$, called also the double cosets, as the formal sum of the different left cosets it contains (more precisely, of the corresponding symbols),

$$
\tau(M)=\sum_{M^{\prime} \in \Gamma_{0}(l) \backslash \Gamma_{0}(l) M \Gamma_{0}(l)}\left(\Gamma_{0}(l) M^{\prime}\right) \quad\left(M \in \Sigma_{0}(l)\right) .
$$

Then each element $T \in \mathcal{H}_{0}(l)$ can also be written as a formal finite linear combination of different left cosets,

$$
T=\sum_{\alpha} c_{\alpha}\left(\Gamma_{0}(l) M_{\alpha}\right) \quad\left(c_{\alpha} \in \mathbb{C}\right)
$$

These linear combinations can be characterized by the condition of invariance with respect to right multiplication by all elements of $\Gamma_{0}(l)$ :

$$
T \sigma=\sum_{\alpha} c_{\alpha}\left(\Gamma_{0}(l) M_{\alpha} \sigma\right)=T \text { for all } \sigma \in \Gamma_{0}(l) .
$$

In this notation, the product in $\mathcal{H}_{0}(l)$ can be defined by

$$
T T^{\prime}=\sum_{\alpha} c_{\alpha}\left(\Gamma_{0}(l) M_{\alpha}\right) \sum_{\beta} c_{\beta}^{\prime}\left(\Gamma_{0}(l) M_{\beta}^{\prime}\right)=\sum_{\alpha, \beta} c_{\alpha} c_{\beta}^{\prime}\left(\Gamma_{0}(l) M_{\alpha} M_{\beta}^{\prime}\right) .
$$


The ring $\mathcal{H}_{0}(l)$ is a commutative $\mathbb{C}$-algebra generated over $\mathbb{C}$ by a countable set of algebraically independent elements. As a set of algebraically independent generators we can take, for example, the double cosets of the form

$$
T(p)=\tau\left(\left(\begin{array}{ll}
1 & 0 \\
0 & p
\end{array}\right)\right), \quad[p]=\tau\left(\left(\begin{array}{ll}
p & 0 \\
0 & p
\end{array}\right)\right),
$$

where $p$ runs over all prime numbers not dividing $l$ (see, e.g., 1 , Theorem 3.3.23]).

In order to define the Hecke operators on theta-functions, we introduce certain linear spaces containing theta-functions. Let $\mathfrak{F}_{m}$ denote the space of all complex-valued realanalytic functions

$$
F=F(z ;(U, V)): \mathbb{H} \times \mathbb{C}^{m} \times \mathbb{C}^{m} \rightarrow \mathbb{C},
$$

where $\mathbb{H}$ is the upper half-plane of the complex variable $z$. For a fixed integral positive definite quadratic form $\mathbf{q}$ in an even number $m$ of variables with matrix $Q$ and a harmonic polynomial $P$ with respect to $\mathbf{q}$, we define an action of the semigroup $\Sigma_{0}(l)$ on the spaces $\mathfrak{F}_{m}$ by the Petersson operators

$$
\Sigma_{0}(l) \ni M: F=\left.F(z ;(U, V)) \mapsto F\right|_{\mathbf{j}} M=j_{Q, P}(M, z)^{-1} F\left(M\langle z\rangle ;(U, V)^{t} M\right),
$$

where

$$
\mathbf{j}=j_{Q, P}\left(\left(\begin{array}{ll}
a & b \\
c & d
\end{array}\right), z\right)=\chi_{\mathbf{q}}(d)(c z+d)^{\frac{m}{2}+g},
$$

$\chi_{\mathbf{q}}$ is character of the quadratic form $\mathbf{q}, g$ is degree of $P$, and

$$
M\langle z\rangle=\left(\begin{array}{ll}
a & b \\
c & d
\end{array}\right)\langle z\rangle=\frac{a z+b}{c z+d} .
$$

It is clear that the function $c z+d$ does not vanish on $\Sigma_{0}(l) \times \mathbb{H}$, so that the same is true for each of the functions $j_{Q, P}(M, z)$. If the matrices $M=\left(\begin{array}{ll}a & b \\ c & d\end{array}\right)$ and $M_{1}=\left(\begin{array}{lll}a_{1} & b_{1} \\ c_{1} & d_{1}\end{array}\right)$ belong to $\Sigma_{0}(l)$ and $M^{\prime}=\left(\begin{array}{cc}a^{\prime} & b^{\prime} \\ c^{\prime} & d^{\prime}\end{array}\right)=M M_{1}$, then an easy and direct computation shows that

and

$$
\left(c \cdot M_{1}\langle z\rangle+d\right)\left(c_{1} z+d_{1}\right)=c^{\prime} z+d^{\prime} \quad(z \in \mathbb{H})
$$

$$
\chi_{\mathbf{q}}\left(d^{\prime}\right)=\chi_{\mathbf{q}}\left(c b_{1}+d d_{1}\right)=\chi_{\mathbf{q}}\left(d d_{1}\right)=\chi_{\mathbf{q}}(d) \chi_{\mathbf{q}}\left(d_{1}\right),
$$

because $c \equiv 0(\bmod l)$. These formulas imply that the functions $j_{Q, P}(M, z)$ satisfy the automorphic factors relations, i.e.,

$$
j_{Q, P}\left(M, M_{1}\langle z\rangle\right) j_{Q, P}\left(M_{1}, z\right)=j_{Q, P}\left(M M_{1}, z\right) \text { for all } M, M_{1} \in \Sigma_{0}(l), z \in \mathbb{H} .
$$

Therefore, the Petersson operators map the space $\mathfrak{F}_{m}$ into itself and satisfy the rule

$$
\left.\left.F\right|_{\mathbf{j}} M\right|_{\mathbf{j}} M_{1}=\left.F\right|_{\mathbf{j}} M M_{1} \quad\left(F \in \mathfrak{F}_{m}, \quad M, M_{1} \in \Sigma_{0}(l)\right) .
$$

This allows us to define the standard representation $\left.T \mapsto\right|_{\mathbf{j}} T$ of the Hecke-Shimura ring $\mathcal{H}_{0}(l)=\mathcal{H}\left(\Gamma_{0}(l), \Sigma_{0}(l)\right)$ on the subspace

$$
\mathfrak{F}_{m}\left(\Gamma_{0}(l)\right)=\left\{F \in \mathfrak{F}_{m}|F|_{\mathbf{j}} \sigma=F \text { for all } \sigma \in \Gamma_{0}(l)\right\}
$$

of all $\Gamma_{0}(l)$-invariant functions by Hecke operators: the Hecke operator $\left.\right|_{\mathbf{j}} T$ on the space $\mathfrak{F}_{m}\left(\Gamma_{0}(l)\right)$ corresponding to an element of the form (3.3) is defined by

$$
\left.F\right|_{\mathbf{j}} T=\left.\sum_{\alpha} c_{\alpha} F\right|_{\mathbf{j}} M_{\alpha} \quad\left(F=F(z ;(U, V)) \in \mathfrak{F}_{m}\left(\Gamma_{0}(l)\right)\right),
$$

where the $\left.\right|_{\mathbf{j}} M_{\alpha}$ are the Petersson operators (3.5) corresponding to $\mathbf{j}=j_{Q, P}(M, z)$. The Hecke operators are independent of the choice of the representatives $M_{\alpha} \in \Gamma_{0}(l) M_{\alpha}$ 
and map the space $\mathfrak{F}_{m}\left(\Gamma_{0}(l)\right)$ into itself. The definition of multiplication in the HeckeShimura rings and relation (3.6) show that Hecke operators satisfy

$$
\left.\left.\right|_{\mathbf{j}} T\right|_{\mathbf{j}} T^{\prime}={ }_{\mathbf{j}} T T^{\prime} \quad \text { for all } T, T^{\prime} \in \mathcal{H}_{0}(l) \text {. }
$$

Hence, the map $\left.T \mapsto\right|_{\mathbf{j}} T$ is a linear representation of the ring $\mathcal{H}_{0}(l)$ on the space $\mathfrak{F}_{m}\left(\Gamma_{0}(l)\right)$. The Hecke operators $(3.8)$ on the space $\mathfrak{F}_{m}\left(\Gamma_{0}(l)\right)$ are called regular Hecke operators.

The functional equations (3.1) show that the theta-function (2.2) of an integral positive definite quadratic form $\mathbf{q}$ of level $l$ in an even number $m$ of variables with matrix $Q$ and a harmonic polynomial $P$ of degree $g$ relative to $\mathbf{q}$, viewed as a function of $z$ and $U, V$, belongs to the space $\mathfrak{F}_{m}\left(\Gamma_{0}(l)\right)$. Thus, this space contains the images of the theta-function under the Hecke operators corresponding to the generators $(3.2)$ of the $\operatorname{ring} \mathcal{H}_{0}(l)$. In particular, this space contains the images of the theta-function under the action of the operators $\mid \mathbf{j} T(p)$ with primes $p$ not dividing $l$, i.e., it contains the functions

$$
\left.\Theta_{P}(z ; Q,(U, V))\right|_{\mathbf{j}} T(p)=\sum_{M \in \Gamma_{0}(l) \backslash \Gamma_{0}(l)\left(\begin{array}{cc}
1 & 0 \\
0 & p
\end{array}\right) \Gamma_{0}(l)} j_{Q, P}(M, z)^{-1} \Theta_{P}\left(M\langle z\rangle ; Q,(U, V)^{t} M\right) .
$$

Since we can take

$$
\Gamma_{0}(l) \backslash \Gamma_{0}(l)\left(\begin{array}{ll}
1 & 0 \\
0 & p
\end{array}\right) \Gamma_{0}(l)=\left\{\left(\begin{array}{ll}
1 & 0 \\
0 & p
\end{array}\right),\left(\begin{array}{ll}
1 & 1 \\
0 & p
\end{array}\right), \ldots,\left(\begin{array}{cc}
1 & p-1 \\
0 & p
\end{array}\right),\left(\begin{array}{ll}
p & 0 \\
0 & 1
\end{array}\right)\right\},
$$

this image can be written in the form

$$
\begin{gathered}
\left.\Theta_{P}(z ; Q,(U, V))\right|_{\mathbf{j}} T(p)=\left(\chi_{\mathbf{q}}(p) p^{k+g}\right)^{-1} \sum_{b=0}^{p-1} \Theta_{P}\left(\frac{z+b}{p} ; Q(U, V)\left(\begin{array}{ll}
1 & 0 \\
b & p
\end{array}\right)\right) \\
\quad+\Theta_{P}\left(p z ; Q,(U, V)\left(\begin{array}{ll}
p & 0 \\
0 & 1
\end{array}\right)\right) \\
=\left(\chi_{\mathbf{q}}(p) p^{k+g}\right)^{-1} \sum_{b=0}^{p-1} \Theta_{P}\left(\frac{z+b}{p} ; Q,(U+b V, p V)\right) \\
+\Theta_{P}(p z ; Q,(p U, V)) .
\end{gathered}
$$

The following particular case of [4, Theorem 6.3] shows that, in some cases, the images of the theta-function under the Hecke operators corresponding to $T(p)$ with primes $p$ can be written as finite linear combinations with constant coefficients of similar thetafunctions.

Theorem 2. Let $\mathbf{q}$ be an integral positive definite quadratic form in an even number $m=2 k$ of variables, let $l$ be the level of $\mathbf{q}$, let $\chi_{\mathbf{q}}$ be the character of $\mathbf{q}$, and let $P$ be a harmonic polynomial of degree $g$ relative to the form $\mathbf{q}$. Then, for each rational prime number $p$ not dividing $l$, the following explicit formulas are valid for the action of the Hecke operator $\left.\right|_{\mathbf{j}} T(p)$ with automorphic factor $\mathbf{j}=j_{Q, P}$ of the form (3.5) on the thetafunction (2.2) of genus 1 with the harmonic coefficient form $P$ and arbitrary parameters $U, V:$ if $\chi_{\mathbf{q}}(p)=1$, then

$$
\left.\Theta_{P}(z ; Q,(U, V))\right|_{\mathbf{j}} T(p)=\frac{\xi(m)}{p^{k-1}} \sum_{D \in A(Q, p) / \Lambda^{m}} \Theta_{P \mid p^{-1} D}\left(z ; p^{-1} Q[D], p D^{-1}(U, V)\right),
$$


where

$$
\begin{aligned}
\xi(m) & = \begin{cases}1 & \text { if } k=1, \\
\prod_{\alpha=1}^{k-1}\left(1+p^{\alpha-1}\right) & \text { if } k>1,\end{cases} \\
A(Q, \mu) & =\left\{D \in \mathbb{Z}_{m}^{m} \mid \mu^{-1} Q[D] \quad \text { is even and } \operatorname{det} \mu^{-1} Q[D]=\operatorname{det} Q\right\}
\end{aligned}
$$

is the set of all automorphs of $Q$ with multiplier $\mu, \Lambda^{m}=G L_{m}(\mathbb{Z})$, and

$$
\left(P \mid p^{-1} D\right)(X)=P\left(p^{-1} D X\right),
$$

but if $\chi_{Q}(p)=-1$ and $k=1$, then

$$
\left.\Theta_{P}(z ; Q,(U, V))\right|_{\mathbf{j}} T(p)=0 .
$$

The obvious relations

$$
\Theta_{P \mid \Lambda}\left(z ; Q[\Lambda], \Lambda^{-1}(U, V)\right)=\Theta_{P}(z ; Q,(U, V)) \text { for each } \Lambda \in \Lambda^{m}=G L_{m}(\mathbb{Z}),
$$

where $(P \mid \Lambda)(X)=P(\Lambda X)$, imply that the sum on the right in (3.10) does not depend on a particular choice of the representatives $D \in A(Q, p) / \Lambda^{m}$.

Generally speaking, the set $A(Q, \mu)$ can be empty, but it is not empty when $\mu=p$ is a prime number satisfying $\chi_{\mathbf{q}}(p)=1$. Clearly, $A(Q, \mu) \Lambda^{m}=A(Q, \mu)$, so that the group $\Lambda^{m}$ operates on each of the sets $A(Q, \mu)$ by right multiplication. Since all automorphs of $A(Q, \mu)$ are integral matrices of fixed determinants $\pm \mu^{m / 2}$, it follows that each set of right classes of automorphs $A(Q, \mu) / \Lambda^{m}$ modulo $\Lambda^{m}$ is always finite.

Standard applications of Hecke operators to Euler factorization of zeta functions of modular forms are based on consideration of common eigenfunctions of the operators. Although Theorem 2 shows that some images of theta-functions under Hecke operators are linear combinations of theta-functions, it gives no direct way to build eigenfunctions from linear combinations of theta-functions. A possible approach can be found in the replacement of the theta-functions with variable parameters $U, V$ by the corresponding theta-series obtained by suitable numerical specializations of the parameters. A version of such a specialization will be discussed in the next section.

\section{§4. ACtion of hereditary Hecke operators on theta-Series, AND EULER PRODUCTS}

Here we consider the action of the regular Hecke-Shimura rings $\mathcal{H}_{0}(l)$ by Hecke operators on theta-functions (2.3) with the specialized parameters $(U, V)$, i.e., on the corresponding theta-series. The resulting hereditary Hecke operators, inherited from the operators (3.8) acting on theta-functions with variable parameters, are in general different from the standard Hecke operators on theta-series viewed only as functions in $z$ ranging in the upper half-plane, because these theta-series may fail to be invariant with respect to the group the form $\Gamma_{0}(l)$. The Hecke operator inherited from an operator $\left.\right|_{\mathbf{j}} T$ will be denoted below by $\left.\right|_{\mathbf{j}} ^{*} T$.

In $\S 2$ we saw that the zeta functions of harmonic theta-functions have good analytic properties. In order to consider another essential feature of arithmetical zeta functions, the Euler product factorization, we follow the classical approach to this problem, initiated by E. Hecke in [5] and based on consideration of eigenfunctions of Hecke operators acting on modular forms. In order to apply Hecke theory, first we need to pass from thetafunctions, which are not modular forms, to suitable modular forms.

Starting with the theta-function (2.2) of an integral positive definite quadratic forms $\mathbf{q}$ in an even number $m=2 k$ of variables of level $l$ with an even matrix $Q$ and with 
harmonic polynomials $P$ of degree $g$ relative to $\mathbf{q}$, we shall specialize the parameters of the theta-function to be rational columns of the form

(4.1) $U=\mathbf{0}, V=l^{-1} L$ with $L \in \mathbb{Z}^{m}$ satisfying the congruence $Q L \equiv \mathbf{0} \quad(\bmod l)$.

Then the theta-function turns into the theta-series

$$
\Theta_{P}(z ; Q \mid L)=\Theta_{P}\left(z ; Q,\left(\mathbf{0}, l^{-1} L\right)\right)=\sum_{N \in \mathbb{Z}^{m}} P\left(N-l^{-1} L\right) e^{\pi i z Q\left[N-l^{-1} L\right]}
$$

with the Fourier expansion

$$
\Theta_{P}(z ; Q \mid L)=\sum_{n=0}^{\infty} r_{Q, P}(n, L) e^{\frac{2 \pi i z n}{l}},
$$

where

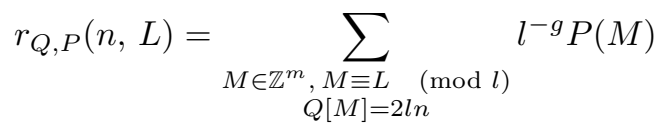

are the Fourier coefficients. Note that the theta-series (1.5) mentioned in $\S 1$ is proportional to the series (4.2) with $\mathbf{q}=8\left(x_{1}^{2}+x_{2}^{2}\right), Q=\left(\begin{array}{cc}16 & 0 \\ 0 & 16\end{array}\right), l=32, L=\left(\begin{array}{c}-8 \\ -8\end{array}\right)$, and $P\left(\left(\begin{array}{l}x_{1} \\ x_{2}\end{array}\right)\right)=x_{2}$.

By formula (3.9) we obtain the following formulas for the action on the theta-series (4.2) of the (hereditary) Hecke operators $\left.\right|_{\mathbf{j}} ^{*} T(p)$ with primes $p$ not dividing the level $l$ of q:

$$
\begin{aligned}
& \left.\Theta_{P}(z ; Q \mid L)\right|_{\mathbf{j}} ^{*} T(p) \\
& =\frac{1}{\chi_{\mathbf{q}}(p) p^{k+g}} \sum_{b=0}^{p-1} \Theta_{P}\left(\frac{z+b}{p} ; Q,\left(l^{-1} b L, l^{-1} p L\right)\right)+\Theta_{P}\left(p z ; Q,\left(\mathbf{0}, l^{-1} L\right)\right) \\
& =\frac{1}{\chi_{\mathbf{q}}(p) p^{k+g}} \sum_{N \in \mathbb{Z}^{m}} P\left(N-l^{-1} p L\right) \sum_{b=0}^{p-1} e^{\pi i\left(\frac{z+b}{p} Q\left[N-l^{-1} p L\right]+2 l^{-1} b^{t} L Q N-l^{-2} b p Q[L]\right)}+\sum_{N \in \mathbb{Z}^{m}} P\left(N-l^{-1} L\right) e^{\pi i p z Q\left[N-l^{-1} L\right]} \\
& =\frac{p}{\chi_{\mathbf{q}}(p) p^{k+g}} \sum_{N \in \mathbb{Z}^{m}, \mathbf{q}(N) \equiv 0}(\bmod p) \\
& +\sum_{N \in \mathbb{Z}^{m}} P\left(N-l^{-1} p L\right) e^{\frac{\pi i z}{p} Q\left[N-l^{-1} p L\right]}
\end{aligned}
$$

Indeed the readily verified identity

$$
\begin{aligned}
& \frac{z+b}{p} Q\left[N-l^{-1} p L\right]+2 l^{-1} b^{t} L Q N-l^{-2} b p Q[L] \\
& \quad=\frac{z}{p} Q\left[N-l^{-1} p L\right]+\frac{b}{p} Q\left[N-l^{-1} p L\right]+2 l^{-1} b^{t} L Q N-l^{-2} b p Q[L] \\
& \quad=\frac{z}{p} Q\left[N-l^{-1} p L\right]+\frac{b}{p} Q[N]=\frac{z}{p} Q\left[N-l^{-1} p L\right]+\frac{2 b}{p} \mathbf{q}(N)
\end{aligned}
$$

shows that

$$
\begin{aligned}
& \sum_{b=0}^{p-1} e^{\pi i\left(\frac{z+b}{p} Q\left[N-l^{-1} p L\right]+2 l^{-1} b^{t} L Q N-l^{-2} b p Q[L]\right)} \\
& \quad=e^{\frac{\pi i z}{p} Q\left[N-l^{-1} p L\right]} \sum_{b=0}^{p-1} e^{\frac{2 \pi i b \mathbf{q}(N)}{p}}=e^{\frac{2 \pi i z}{p} Q\left[N-l^{-1} p L\right]} \times \begin{cases}p & \text { if } p \mid \mathbf{q}(N), \\
0 & \text { if } p \nmid \mathbf{q}(N) .\end{cases}
\end{aligned}
$$


Writing the series (4.5) as a Fourier series, in the notation (4.4) we get the formula

$$
\left.\Theta_{P}(z ; Q \mid L)\right|_{\mathbf{j}} ^{*} T(p)=\sum_{n=0}^{\infty}\left(\frac{1}{\chi_{\mathbf{q}}(p) p^{k-1+g}} r_{Q, P}(n p, p L)+r_{Q, P}(n / p, L)\right) e^{\frac{2 \pi i z n}{l}}
$$

where the second term in the parenthesis is present only if $p$ divides $n$.

Now we consider a linear combination of the theta-series (4.2) written in the form (4.3) with constant coefficients $\Phi(L)$ depending on $L$ modulo l, i.e., the function

$$
\begin{aligned}
F=\Theta_{\Phi, P}(z ; Q)= & \sum_{\substack{L \in \mathbb{Z}^{m} / l \mathbb{Z}^{m}, Q L \equiv \mathbf{0}(\bmod l)}} \Phi(L) \Theta_{P}(z ; Q \mid L) \\
= & \sum_{n=0}^{\infty}\left(\sum_{\substack{L \in \mathbb{Z}^{m} / l \mathbb{Z}^{m}, Q L \equiv \mathbf{0}(\bmod l)}} \Phi(L) r_{Q, P}(n, L)\right) e^{\frac{2 \pi i z n}{l}}
\end{aligned}
$$

with the Fourier coefficients

$$
r_{Q, P}(n, \Phi)=\sum_{\substack{L \in \mathbb{Z}^{m} / l \mathbb{Z}^{m}, Q L \equiv \mathbf{0}(\bmod l)}} \Phi(L) r_{Q, P}(n, L)=\sum_{\substack{M \in \mathbb{Z}^{m}, Q[M]=2 n l \\ Q M \equiv \mathbf{0}(\bmod h)}} l^{-g} \Phi(M) P(M) .
$$

Using (4.6), we obtain

$$
\begin{gathered}
\left.F\right|_{\mathbf{j}} ^{*} T(p)=\left.\Theta_{\Phi, P}(z ; Q)\right|_{\mathbf{j}} ^{*} T(p)=\left.\sum_{\substack{L \in \mathbb{Z}^{m} / l \mathbb{Z}^{m}, Q L \equiv \mathbf{0}(\bmod l)}} \Phi(L) \Theta_{P}(z ; Q \mid L)\right|_{\mathbf{j}} ^{*} T(p) \\
=\sum_{\substack{L \in \mathbb{Z}^{m} / l \mathbb{Z}^{m}, Q L \equiv \mathbf{0}(\bmod l)}} \Phi(L) \sum_{n=0}^{\infty}\left(\frac{1}{\chi_{\mathbf{q}}(p) p^{k-1+g}} r_{Q, P}(n p, p L)+r_{Q, P}(n / p, L)\right) e^{\frac{2 \pi i z n}{l}} \\
=\frac{1}{\chi_{\mathbf{q}}(p) p^{k-1+g}} \sum_{n=0}^{\infty} \sum_{\substack{L \in \mathbb{Z}^{m} / l \mathbb{Z}^{m}, Q L \equiv \mathbf{0}(\bmod l)}} \Phi(L) r_{Q, P}(n p, p L) e^{\frac{2 \pi i z n}{l}} \\
+\sum_{n=0}^{\infty} r_{Q, P}(n / p, \Phi) e^{\frac{2 \pi i z n}{l}} .
\end{gathered}
$$

In order to reshape the first sum to a convenient form, now we assume that the coefficient function $\Phi$ satisfies a homogeneity condition of the form

$$
\Phi(a L)=\phi(a) \Phi(L) \quad \text { for all } a \in \mathbb{Z} \quad \text { with } \operatorname{gcd}(a, l)=1,
$$

with a function $\phi: \mathbb{Z} / l \mathbb{Z} \rightarrow \mathbb{C}$. If $\Phi$ is not identically zero, it is easily seen that the function $\phi$ must satisfy the conditions

$$
\phi(1)=1, \quad \phi(a b)=\phi(a) \phi(b) \quad \text { if } a \text { and } b \text { are prime to } l
$$

in particular, the value $\phi(a)$, where $a$ is prime to $l$, must be a root of unity.

Returning to (4.9), we observe that if $\Phi$ satisfies condition (4.10), then, since $p$ does not divide $l$, we can replace the sum over $L$ by the sum over $p^{\prime} L$ with $p^{\prime}$ satisfying 
$p^{\prime} p \equiv 1(\bmod l)$, obtaining

$$
\begin{aligned}
& \sum_{\substack{L \in \mathbb{Z}^{m} / l \mathbb{Z}^{m}, Q L \equiv \mathbf{0}(\bmod l)}} \Phi(L) r_{Q, P}(n p, p L)=\sum_{\substack{L \in \mathbb{Z}^{m} / l \mathbb{Z}^{m} \\
Q L \equiv \mathbf{0}(\bmod l)}} \Phi\left(p^{\prime} L\right) r_{Q, P}\left(n p, p^{\prime} p L\right) \\
& =\phi\left(p^{\prime}\right) \sum_{\substack{L \in \mathbb{Z}^{m} / l \mathbb{Z}^{m} \\
Q L \equiv \mathbf{0}(\bmod l)}} \Phi(L) r_{Q, P}(n p, L)=\bar{\phi}(p) r_{Q, P}(n p, \Phi),
\end{aligned}
$$

because the number $\phi\left(p^{\prime}\right)=\phi(p)^{-1}=\bar{\phi}(p)$ is complex conjugate to $\phi(p)$. Substituting the last expression in (4.9), finally we get the formula

$$
\begin{aligned}
\left.F\right|_{\mathbf{j}} ^{*} T(p) & =\left.\Theta_{\Phi, P}(z ; Q)\right|_{\mathbf{j}} ^{*} T(p) \\
& =\sum_{n=0}^{\infty}\left(\frac{\bar{\phi}(p)}{\chi_{\mathbf{q}}(p) p^{k-1+g}} r_{Q, P}(n p, \Phi)+r_{Q, P}(n / p, \Phi)\right) e^{\frac{2 \pi i z n}{l}} .
\end{aligned}
$$

Suppose now that the function $F=\Theta_{\Phi, P}(z ; Q)$ of the form (4.7) is an eigenfunction for the operator $\left.\right|_{\mathbf{j}} ^{*} T(p)$ with the eigenvalue $\lambda_{F}(p)$. Then, by (4.12), the Fourier coefficients (4.8) of $F$ satisfy the relations

$$
\lambda_{F_{\Phi}}(p) r_{Q, P}(n, \Phi)=\frac{\bar{\phi}(p)}{\chi_{\mathbf{q}}(p) p^{k-1+g}} r_{Q, P}(n p, \Phi)+r_{Q, P}(n / p, \Phi),
$$

provided that the function $\Phi$ satisfies condition (4.10).

Relations (4.13) with numbers $n \neq 0$ differing by powers of a fixed prime $p$ can be viewed as recurrence relations; they can be used for summing formal power series of the form

$$
R_{p, n}(t)=\sum_{\delta=0}^{\infty} r_{Q, P}\left(p^{\delta} n, \Phi\right) t^{\delta}
$$

Suppose that $n$ is not divisible by $p$; then by (4.12) we have

$$
\begin{aligned}
\lambda_{F}(p) R_{p, n}(t) & =\frac{\bar{\phi}(p)}{\chi_{\mathbf{q}}(p) p^{k-1+g}} \sum_{\delta=0}^{\infty} r_{Q, P}\left(p^{\delta+1} n, \Phi\right) t^{\delta}+\sum_{\delta=1}^{\infty} r_{Q, P}\left(p^{\delta-1} n, \Phi\right) t^{\delta} \\
& =\frac{\bar{\phi}(p)}{\chi_{\mathbf{q}}(p) p^{k-1+g t}}\left(R_{p, n}(t)-r_{Q, P}(n, \Phi)\right)+t R_{p, n}(t),
\end{aligned}
$$

whence

$$
\begin{aligned}
R_{p, n}(t) & =\sum_{\delta=0}^{\infty} r_{Q, P}\left(p^{\delta} n, \Phi\right) t^{\delta} \\
& =\left(1-p^{k-1+g} \chi_{\mathbf{q}}(p) \phi(p) \lambda_{F}(p) t+p^{k-1+g} \chi_{\mathbf{q}}(p) \phi(p) t^{2}\right)^{-1} r_{Q, P}(n, \Phi) .
\end{aligned}
$$

These summation formulas will be used below for Euler product factorization of the zetafunctions corresponding to the eigenfunctions of Hecke operators on spaces spanned by theta-series (4.2).

We recall that the zeta-function (2.4) of the theta-series (4.2) is defined by the Dirichlet series

$$
\zeta_{P}(s ; Q \mid L)=\sum_{N \in \mathbb{Z}^{m}, N \neq l^{-1} L} \frac{P\left(N-l^{-1} L\right)}{\mathbf{q}\left(N-l^{-1} L\right)^{s+\frac{g}{2}}}=\sum_{n=1}^{\infty} \frac{r_{Q, P}(n, L)}{(n / l)^{s+\frac{g}{2}}}
$$


where the $r_{Q, P}(n, L)$ are the Fourier coefficients (4.4) of the theta-series. Hence, the zeta-function of the linear combination (4.7) has the form

$$
\zeta(s, F)=\sum_{\substack{L \in \mathbb{Z}^{m} / l \mathbb{Z}^{m}, Q L \equiv \mathbf{0}(\bmod l)}} \Phi(L) \zeta_{P}(s ; Q \mid L)=\sum_{n=1}^{\infty} \frac{l^{s+\frac{g}{2}} r_{Q, P}(n, \Phi)}{n^{s+\frac{g}{2}}},
$$

where the $r_{Q, P}(n, \Phi)$ are the Fourier coefficients (4.8) of $F$. This zeta-function together with the zeta-functions (4.15) converges absolutely for $\operatorname{Re} s>k=m / 2$, and uniformly in every half-plane $\operatorname{Re} s>k+\epsilon$ with $\epsilon>0$. Thus, $\zeta(s, F)$ is an analytic function for $\operatorname{Re} s>k$.

Suppose that the function $F$ is an eigenfunction for the operator $\left.\right|_{\mathbf{j}} ^{*} T(p)$ with the eigenvalue $\lambda_{F}(p)$, where $p$ is a prime number not dividing $l$. Then, using identity (4.14), we can factor the function $\zeta(s, F)$ in the half-plane $\operatorname{Re} s>k$ as follows:

$$
\begin{aligned}
\zeta(s, F) & \left.=\sum_{\delta=0}^{\infty} \sum_{n \geq 1, n \neq 0} \frac{l^{s+\frac{g}{2}} r_{Q, P}\left(p^{\delta} n, \Phi\right)}{\left(p^{\delta} n\right)^{s+\frac{g}{2}}} p\right) \\
& =\left(1-\frac{p^{k-1+\frac{g}{2}} \chi_{\mathbf{q}}(p) \phi(p) \lambda_{F}(p)}{p^{s}}+\frac{p^{k-1} \chi_{\mathbf{q}}(p) \phi(p)}{p^{2 s}}\right)^{-1} \sum_{n \neq 0} \frac{l_{(\bmod p)}^{s+\frac{g}{2}} r_{Q, P}(n, \Phi)}{n^{s+\frac{g}{2}}} .
\end{aligned}
$$

Assuming that $F$ is an eigenfunction for all operators $\left.\right|_{\mathbf{j}} ^{*} T(p)$ with prime $p$ not dividing $l$ and applying the last relation to each of the primes, we arrive at the following statement.

Theorem 3. Suppose that a linear combination F (like in (4.7)) of theta-series for an integral positive definite quadratic form $\mathbf{q}$ of level $l$ in an even number $m=2 k$ of variables with a harmonic polynomial $P$ of degree $g$, where the coefficients $\Phi$ satisfy condition (4.10), is a common eigenfunction of the (hereditary) Hecke operators $\left.\right|_{\mathbf{j}} ^{*} T(p)$ for all prime numbers $p$ not dividing $l$ with eigenvalues $\lambda_{F}(p)$. Then the zeta function $\zeta(s, F)$ of $F$ admits factorization into an Euler product convergent absolutely and uniformly in every half-plane $\operatorname{Re} s>k+\epsilon$ with $\epsilon>0$; this Euler product looks like this:

$$
\begin{aligned}
\zeta(s, F) & =\sum_{n=1}^{\infty} \frac{l^{s+\frac{g}{2}} r_{Q, P}(n, \Phi)}{n^{s+\frac{g}{2}}} \\
& =\prod_{p \in \mathbb{P}, p \nmid l}\left(1-\frac{p^{k-1+\frac{g}{2}} \chi_{\mathbf{q}}(p) \phi(p) \lambda_{F}(p)}{p^{s}}+\frac{p^{k-1} \chi_{\mathbf{q}}(p) \phi(p)}{p^{2 s}}\right)^{-1} \sum_{n \mid l \infty} \frac{l^{s+\frac{g}{2}} r_{Q, P}(n ; \Phi)}{n^{s+\frac{g}{2}}},
\end{aligned}
$$

where $\mathbb{P}$ is the set of all positive rational prime numbers, and the notation $n \mid l^{\infty}$ means that $n$ divides a power of $l$, i.e., each prime divisor of $n$ divides $l$.

\section{§5. EIGENFUNCTIONS FOR THETA-SERIES OF MULTIPLES OF THE SUM OF TWO SQUARES}

In accordance with Theorem 3, for applications of Hecke operators to Euler factorizations of the zeta-functions of theta-series one needs to build common eigenfunction of the operators and find the corresponding eigenvalues. In the case of theta-series of one variable, so far the only way to approach this problem is to use explicit formulas, like formulas of Theorem 2, which express the images of the theta-series under the Hecke operators in terms of similar theta-series. In this section we apply this approach to the simplest case of harmonic theta-series of binary quadratic forms proportional to the sum of two squares. The general case of positive definite integral binary forms is a question for the future. 
Here we consider the action of (hereditary) Hecke operators on the harmonic thetaseries (4.2) of a quadratic form

$$
\mathbf{q}(X)=t\left(x_{1}^{2}+x_{2}^{2}\right) \quad(t=1,2, \ldots) \quad \text { with the matrix } Q=\left(\begin{array}{cc}
2 t & 0 \\
0 & 2 t
\end{array}\right)
$$

of level $l=l(Q)=4 t$, where a column $L \in \mathbb{Z}^{2}$ satisfies $Q L \equiv 0(\bmod l)$, that is $L \equiv 0$ $(\bmod 2)$, so that $L=-2 T$ with $T \in \mathbb{Z} / 2 t \mathbb{Z}$, and where the harmonic polynomial $P$ is one of the binomials

$$
P^{ \pm}(X)=((1, \pm i) X)^{g}=\left(x_{1} \pm i x_{2}\right)^{g} \quad\left(X=\left(\begin{array}{l}
x_{1} \\
x_{2}
\end{array}\right)\right) .
$$

The character $\chi_{\mathbf{q}}$ of the form $\mathbf{q}$ is defined on the prime numbers by the conditions $\chi_{\mathbf{q}}(p)=0$ if $p \mid l$ and $\chi_{\mathbf{q}}(p)=(-1)^{\frac{p-1}{2}}$ otherwise.

Each theta-series of this sort looks like this:

$$
\Theta_{P}(z ; Q \mid-2 T)=\sum_{N \in \mathbb{Z}^{m}} P\left(N+(2 t)^{-1} T\right) e^{\pi i z Q\left[N+(2 t)^{-1} T\right]} .
$$

In the notation and under the assumptions of Theorem 2, for the theta-series (5.1) and a prime $p$ not dividing the level $l=4 t$, in the case where $\chi_{\mathbf{q}}(p)=1$, i.e., if $p \equiv 1(\bmod 4)$, we have the identity

$$
\left.\Theta_{P}(z ; Q \mid-2 T)\right|_{\mathbf{j}} ^{*} T(p)=\sum_{D \in A(Q, p) / \Lambda^{2}} \Theta_{P \mid p^{-1} D}\left(z ; p^{-1} Q[D] \mid-2 p D^{-1} T\right),
$$

and if $\chi_{\mathbf{q}}(p)=-1$, i.e., $p \equiv 3(\bmod 4)$, then

$$
\left.\Theta_{P}(z ; Q \mid-2 T)\right|_{\mathbf{j}} ^{*} T(p)=0 .
$$

Thus, when looking for eigenfunctions, it suffices to consider only the case where $p \equiv 1$ $(\bmod 4)$. Note that in this case each right $\operatorname{coset} D \Lambda^{2} \in A(Q, p)$ contains a representative $D^{\prime}$ with positive determinant, and such a representative is unique up to a right factor belonging to the modular group $\Gamma=S L_{2}(\mathbb{Z})$. Then it follows easily that the mapping $D \Lambda^{2} \mapsto D^{\prime} \Gamma$ determines a bijection of the sets of the classes $A(Q, p) / \Lambda^{2}$ and $A^{+}(Q, p) / \Gamma$, where $A^{+}(Q, \mu)=\{D \in A(Q, \mu) \mid \operatorname{det} D>0\}$ is the set of all proper automorphs of $Q$ with multiplier $\mu$. Thus, we can rewrite formula (5.2) in the form

$$
\begin{aligned}
& \left.\Theta_{P}(z ; Q \mid-2 T)\right|_{\mathbf{j}} ^{*} T(p)=\sum_{D \in A^{+}(Q, p) / \Gamma} \Theta_{P \mid p^{-1} D}\left(z ; p^{-1} Q[D] \mid-2 p D^{-1} T\right) \\
& =\sum_{N \in \mathbb{Z}^{2}} \sum_{D \in A^{+}(Q, p) / \Gamma} P\left(p^{-1} D\left(N+(2 t)^{-1} p D^{-1} T\right)\right) e^{\pi i z p^{-1} Q[D]\left[N+(2 t)^{-1} p D^{-1} T\right]} .
\end{aligned}
$$

Since $p$ does not divide $l$, it easily follows that for each $D \in A^{+}(Q, p)$ the matrix $p^{-1} Q[D]$ has the same divisor $t$ as the matrix $Q$; hence, it is of the form $t Q^{\prime}$, where $Q^{\prime}$ is an even primitive matrix of determinant $\operatorname{det} Q^{\prime}=\operatorname{det} Q / t^{2}=4$, which, therefore, has the form $Q^{\prime}={ }^{t} U\left(\begin{array}{ll}2 & 0 \\ 0 & 2\end{array}\right) U$ with $U \in \Gamma$ (the class number of the sum of two squares is one). Thus, the representatives $D$ of the classes $A^{+}(Q, p) / \Gamma$ can be chosen so that $p^{-1} Q[D]=Q$ and the sum over $A^{+}(Q, p) / \Gamma$ can be replaced by the sum over the set $R^{+}(Q, p Q) / E^{+}$, where

$$
R^{+}(Q, p Q)=\left\{D \in \mathbb{Z}_{2}^{2} \mid \operatorname{det} D>0, Q[D]=p Q\right\}
$$

is the set of proper representations of $p Q$ by $Q$, and

$$
E^{+}=\{U \in \Gamma \mid Q[U]=Q\}=\left\{\left(\begin{array}{ll}
1 & 0 \\
0 & 1
\end{array}\right),\left(\begin{array}{cc}
-1 & 0 \\
0 & -1
\end{array}\right)\left(\begin{array}{cc}
0 & 1 \\
-1 & 0
\end{array}\right),\left(\begin{array}{cc}
0 & -1 \\
1 & 0
\end{array}\right)\right\}
$$


is the group of the proper units of $Q$. Thus, we can rewrite (5.4) as follows:

$$
\begin{aligned}
& \left.\Theta_{P}(z ; Q \mid-2 T)\right|_{\mathbf{j}} ^{*} T(p) \\
& \quad=\sum_{N \in \mathbb{Z}^{2}} \sum_{D \in R^{+}(Q, p Q) / E^{+}} P\left(p^{-1} D\left(N+(2 t)^{-1} p D^{-1} T\right)\right) e^{\pi i z Q\left[N+(2 t)^{-1} p D^{-1} T\right]} .
\end{aligned}
$$

Since $p \equiv 1(\bmod 4)$, it is easily seen that the set $R^{+}(Q, p Q)$ can be taken in the form

$$
R^{+}(Q, p Q)=\left\{\left(\begin{array}{cc}
a & b \\
-b & a
\end{array}\right) \mid a, b \in \mathbb{Z}, a^{2}+b^{2}=p\right\}
$$

and consists of 8 matrices, while a set of representatives $R^{+}(Q, p Q) / E^{+}$can be taken, e.g., in the form

$$
\begin{aligned}
R^{+}(Q, p Q) / E^{+} & =\left\{\left(\begin{array}{cc}
a & b \\
-b & a
\end{array}\right),\left(\begin{array}{cc}
a & -b \\
b & a
\end{array}\right) \mid a, b \in \mathbb{Z}, a^{2}+b^{2}=p, 0<a<b\right\} \\
& =\left\{D_{1}, D_{2}\right\}
\end{aligned}
$$

and consists of 2 matrices. Now, consider the action of the operator $\left.\right|_{\mathbf{j}} ^{*} T(p)$ on the linear combination of theta-series (5.1) with a coefficient function $\Phi$, which we shall write in the form

$$
\Phi(T)=\varphi\left(t_{1}+i t_{2}\right) \quad\left(T=\left(\begin{array}{c}
t_{1} \\
t_{2}
\end{array}\right) \in T \in \mathbb{Z}^{2} / 2 t \mathbb{Z}^{2}, t_{1}+i t_{2} \in \mathcal{O}=\mathbb{Z}[i] / 2 t \mathcal{O}\right)
$$

with, for example, $P(X)=P^{+}(X)=\left(x_{1}+i x_{2}\right)^{g}$, i.e., we consider the action of the above operator on the theta-series

$$
\begin{aligned}
F & =\sum_{T \in \mathbb{Z}^{2} / 2 t \mathbb{Z}^{2}} \Phi(T) \Theta_{P}(z ; Q \mid-2 T) \\
& =\sum_{N \in \mathbb{Z}^{2}} \sum_{T \in \mathbb{Z}^{2} / 2 t \mathbb{Z}^{2}} \Phi(T) P\left(N+(2 t)^{-1} T\right) e^{2 \pi i z Q\left[N+(2 t)^{-1} T\right]} \\
& =(2 t)^{-g} \sum_{\substack{n_{1}, n_{2} \in \mathbb{Z}, t_{1}, t_{2} \in \mathbb{Z} / 2 t \mathbb{Z}}} \varphi\left(t_{1}+i t_{2}\right)\left(\left(2 t n_{1}+t_{1}\right)+i\left(2 t n_{2}+t_{2}\right)\right)^{g} e^{\frac{\pi i z}{2 t}\left(\left(2 t n_{1}+t_{1}\right)^{2}+\left(2 t n_{2}+t_{2}\right)^{2}\right)} \\
& =(2 t)^{-g} \sum_{m_{1}, m_{2} \in \mathbb{Z}} \varphi\left(m_{1}+i m_{2}\right)\left(m_{1}+i m_{2}\right)^{g} e^{\frac{\pi i z}{2 t}\left(m_{1}^{2}+m_{2}^{2}\right)} .
\end{aligned}
$$

Using formulas (5.5), we obtain

$$
\begin{aligned}
& \left.F\right|_{\mathbf{j}} ^{*} T(p)=\left.\left(\sum_{T \in \mathbb{Z}^{2} / 2 t \mathbb{Z}^{2}} \Phi(T) \Theta_{P}(z ; Q \mid-2 T)\right)\right|_{\mathbf{j}} ^{*} T(p) \\
& =\sum_{N \in \mathbb{Z}^{2}} \sum_{D \in R^{+}(Q, p Q) / E^{+}} \sum_{T \in \mathbb{Z}^{2} / 2 t \mathbb{Z}^{2}} \Phi(T) P\left(p^{-1} D\left(N+(2 t)^{-1} p D^{-1} T\right)\right) e^{\pi i z Q\left[N+(2 t)^{-1} p D^{-1} T\right]} \\
& =\sum_{N \in \mathbb{Z}^{2}} \sum_{D \in R^{+}(Q, p Q) / E^{+}} \sum_{T \in \mathbb{Z}^{2} / 2 t \mathbb{Z}^{2}} \Phi\left(p^{\prime} D T\right) P\left(p^{-1} D\left(N+(2 t)^{-1} T\right)\right) e^{\pi i z Q\left[N+(2 t)^{-1} T\right]},
\end{aligned}
$$

where at the last step we have replaced $T$ by $p^{\prime} D T$ with an inverse $p^{\prime}$ of $p$ modulo $2 t$. Using the notation (5.8) for $D=D_{1}=\left(\begin{array}{cc}a & b \\ -b & a\end{array}\right), D_{2}=\left(\begin{array}{cc}a & -b \\ b & a\end{array}\right)$ in the set of representatives (5.7), we have

$$
\begin{aligned}
\Phi\left(p^{\prime} D_{1} T\right) & =\Phi\left(p^{\prime}\left(\begin{array}{cc}
a & b \\
-b & a
\end{array}\right)\left(\begin{array}{l}
t_{1} \\
t_{2}
\end{array}\right)\right)=\Phi\left(p^{\prime}\left(\begin{array}{c}
a t_{1}+b t_{2} \\
-b t_{1}+a t_{2}
\end{array}\right)\right) \\
& =\varphi\left(p^{\prime}\left(a t_{1}+b t_{2}+i\left(-b t_{1}+a t_{2}\right)\right)\right)=\varphi\left(p^{\prime}(a+i b)\left(t_{1}+i t_{2}\right)\right)
\end{aligned}
$$


and

$$
\begin{aligned}
\Phi\left(p^{\prime} D_{2} T\right) & =\Phi\left(p^{\prime}\left(\begin{array}{cc}
a & -b \\
b & a
\end{array}\right)\left(\begin{array}{l}
t_{1} \\
t_{2}
\end{array}\right)\right)=\Phi\left(p^{\prime}\left(\begin{array}{l}
a t_{1}-b t_{2} \\
b t_{1}+a t_{2}
\end{array}\right)\right) \\
& =\varphi\left(p^{\prime}\left(a t_{1}-b t_{2}+i\left(b t_{1}+a t_{2}\right)\right)\right)=\varphi\left(p^{\prime}(a-i b)\left(t_{1}+i t_{2}\right)\right) .
\end{aligned}
$$

Now, suppose that the function $\varphi$ is multiplicative, i.e.,

$$
\varphi(\alpha \beta)=\varphi(\alpha) \varphi(\beta) \quad(\alpha, \beta \in \mathcal{O} / 2 l \mathcal{O}),
$$

in particular, $\varphi(1)=1$ if $\varphi$ is not identically zero. Then the above formulas imply the relations

$$
\Phi\left(p^{\prime} D_{1} T\right)=\varphi\left(p^{\prime}(a+i b)\left(t_{1}+i t_{2}\right)\right)=\varphi\left(p^{\prime}(a+i b)\right) \Phi(T)
$$

and

$$
\Phi\left(p^{\prime} D_{2} T\right)=\varphi\left(p^{\prime}(a-i b)\left(t_{1}+i t_{2}\right)\right)=\varphi\left(p^{\prime}(a-i b)\right) \Phi(T) .
$$

On the other hand, for, e.g., the harmonic polynomial $P=P^{+}(X)=((1, i) X)^{g}$, we have

$$
\begin{aligned}
P\left(p^{-1} D_{1} X\right) & =\left((1, i) p^{-1} D_{1} X\right)^{g}=\left(p^{-1}(a-i b, b+i a) X\right)^{g} \\
& =\left(p^{-1}(a-i b)(1, i) X\right)^{g}=\left(p^{-1}(a-i b)\right)^{g} P(X),
\end{aligned}
$$

and similarly,

$$
P\left(p^{-1} D_{2} X\right)=\left(p^{-1}(a+i b)\right)^{g} P(X) .
$$

Hence, if the coefficient function (5.8) satisfies the multiplicativity condition (5.11), then formula (5.10) for, say, $P=P^{+}$can be rewritten as follows:

$$
\begin{aligned}
\left.F\right|_{\mathbf{j}} ^{*} T(p) & =\sum_{N \in \mathbb{Z}^{2}} \sum_{T \in \mathbb{Z}^{2} / 2 t \mathbb{Z}^{2}} \Phi\left(p^{\prime} D_{1} T\right) P\left(p^{-1} D_{1}\left(N+(2 t)^{-1} T\right)\right) e^{\pi i z Q\left[N+(2 t)^{-1} T\right]} \\
& \quad+\sum_{N \in \mathbb{Z}^{2}} \sum_{T \in \mathbb{Z}^{2} / 2 t \mathbb{Z}^{2}} \Phi\left(p^{\prime} D_{2} T\right) P\left(p^{-1} D_{2}\left(N+(2 t)^{-1} T\right)\right) e^{\pi i z Q\left[N+(2 t)^{-1} T\right]} \\
= & \sum_{N \in \mathbb{Z}^{2}} \sum_{T \in \mathbb{Z}^{2} / 2 t \mathbb{Z}^{2}}\left(\varphi\left(p^{\prime}(a+i b)\right)\left(p^{-1}(a-i b)\right)^{g}+\varphi\left(p^{\prime}(a-i b)\right)\left(p^{-1}(a+i b)\right)^{g}\right) \\
= & \frac{\varphi\left(p^{\prime}\right)}{p^{g}}\left(\varphi(a+i b)(a-i b)^{g}+\varphi(a-i b)(a+i b)^{g}\right) F=\lambda_{F}(p) F,
\end{aligned}
$$

i.e., the linear combination $F$ is an eigenfunction of the operator $\left.\right|_{\mathbf{j}} ^{*} T(p)$ with the eigenvalue

$$
\lambda_{F}(p)=\frac{\varphi\left(p^{\prime}\right)}{p^{g}}\left(\varphi(a+i b)(a-i b)^{g}+\varphi(a-i b)(a+i b)^{g}\right) .
$$

The above consideration allows us to apply Theorem 3 to the zeta-function

$$
\zeta(s, F)=\sum_{\substack{N \in \mathbb{Z}^{2}, T \in \mathbb{Z}^{2} / 2 t \mathbb{Z}^{2}, N+(2 t)^{-1} T \neq \mathbf{0}}} \frac{\Phi(T) P\left(N+(2 t)^{-1} T\right)}{\mathbf{q}\left(N+(2 t)^{-1} T\right)^{s+\frac{g}{2}}}=\sum_{n=1}^{\infty} \frac{c_{F}(n)}{n^{s+\frac{g}{2}}}
$$

of the linear combination (5.9) in the case of harmonic polynomials $P=P^{ \pm}$, because, clearly, the coefficient function $\Phi$ satisfies condition (4.10), where the function $\phi: \mathbb{Z} / 2 t \mathbb{Z}$ is equal to the restriction to $\mathbb{Z} \subset \mathbb{Z}[i]$ of the function $\varphi$. Then we see that the zeta-function 
(5.14) admits factorization in an Euler product absolutely and uniformly convergent in every half-plane $\operatorname{Re} s>1+\epsilon$ with $\epsilon>0$; this Euler product looks like this:

$$
\zeta(s, F)=\prod_{p \in \mathbb{P}, p \nmid 2 t}\left(1-\frac{p^{\frac{g}{2}} \chi_{\mathbf{q}}(p) \varphi(p) \lambda_{F}(p)}{p^{s}}+\frac{\chi_{\mathbf{q}}(p) \varphi(p)}{p^{2 s}}\right)^{-1} \sum_{n \mid(2 t) \infty} \frac{c_{F}(n)}{n^{s+\frac{g}{2}}},
$$

where $\lambda_{F}(p)$ is the eigenvalue $(5.13)$ if $p \equiv 1(\bmod 4)$, and $\lambda_{F}(p)=0$ if $p \equiv-1(\bmod 4)$.

\section{ACKNowledgment}

I am much grateful to my colleagues and the administration of Max-Planck-Institut für Mathematik (Bonn, Germany), where the present work was completed and discussed during the Spring of 2010, for their kind invitation and excellent stimulating atmosphere.

\section{REFERENCES}

[1] A. N. Andrianov, Quadratic forms and Hecke operators, Grundlehren Math. Wiss., Bd. 286, Springer-Verlag, Berlin, 1987. MR0884891 (88g:11028)

[2] Symmetries of harmonic theta functions of integer-valued quadratic forms, Uspekhi Mat. Nauk 50 (1995), no. 4, 3-44; English transl., Russian Math. Surveys 50 (1995), no. 4, 661-700. MR.1357882(96i:11041)

[3] _ Harmonic theta-functions and Hecke operators, Algebra i Analiz 8 (1996), no. 5, 1-31; English transl., St. Petersburg Math. J. 8 (1997), no. 5, 695-720. MR1428987 (98a:11053)

[4] Interaction sums and action of Hecke operators on theta-series, Acta Arith. 140 (2009), no. 3, 271-304. MR2564466 (2011c:11080)

[5] E. Hecke, Über Modulfunktionen und die Dirichletschen Reihen mit Eulerscher Produktentwicklung. I, II, Math. Ann. 114 (1937), 1-28, 316-351 (=Math. Werke, Vandenhoeck and Ruprecht, Göttingen, 1959, pp. 644-707). MR1513122; MR 1513142

[6] A. Ogg, Modular forms and Dirichlet series, W. A. Benjamin, Inc., New York-Amsterdam, 1969. MR0256993 (41:1648)

[7] B. Schoeneberg, Das Verhalten von mehrfachen Thetareihen bei Modulsubstitutionen, Math. Ann. 116 (1939), no. 1, 511-523, 780. MR 1513241 MR1513260

[8] C. L. Siegel, Lectures on advanced analytic number theory, Tata Inst. Fund. Res. Lectures on Math., No. 23, Tata Inst. Fund. Res., Bombay, 1961 (Reissued, 1965). MR0262150 (41:6760)

[9] I. M. Vinogradov, Elements of number theory, GITTL, Moscow-Leningrad, 1952; English transl., Dover Publ., Inc., New York, 1954. MR0062138 (15:933e)

[10] A. Weil, Sur les courbes algébriques et les variétés qui s'en déduisent, Actualités Sci. Ind., No. 1041, Hermann, Paris, 1948. MR0027151 (10:262c)

St. Petersburg Branch, Steklov Mathematical Institute, Fontanka 27, St. Petersburg 191023, RUSSIA

E-mail address: anatoli.andrianov@gmail.com

Current address: Max-Planck-Institut für Mathematik, Vivatsgasse 7, 53111 Bonn, Germany

E-mail address: andriano@mpim-bonn.mpg.de

Received 12/OCT/2010

Translated by THE AUTHOR 